\title{
Sensory-specific associations in flavor-preference reversal learning
}

\author{
Janina Scarlet, Vincent Campese, and Andrew R. Delamater \\ Brooklyn College, CUNY, Brooklyn, New York
}

\begin{abstract}
In two experiments, we examined the effect of reversal learning on the status of initially learned associations. In Experiment 1, thirsty rats were first taught to associate one flavor with sucrose and another flavor with Polycose. These relations were then reversed in a subsequent phase. One of the nutrients was then devalued by being paired with lithium chloride. The results of a two-bottle flavor-choice test revealed that the most recently learned associations governed performance. In Experiment 2, we aimed to discern whether the initially learned associations in Experiment 1 were weakened or masked by reversal learning. In order to address this question, either a 1-day (Group Immediate) or a 21-day (Group Delayed) retention interval was interpolated between the reversal and devaluation phases. Subsequent flavor-choice tests revealed that Group Immediate avoided the flavor most recently associated with the devalued nutrient but that Group Delayed avoided the flavor that was initially associated with the devalued nutrient. These findings suggest that the second-learned associations do not erase, but transiently mask, the first-learned associations, which subsequently recover over a retention interval. These results suggest a parallel in the mechanisms of extinction and reversal learning.
\end{abstract}

Robust conditioned flavor preferences can be established by pairing a flavor conditioned stimulus (CS) with a nutrient unconditioned stimulus (US). Preferences for the flavor cue are acquired both when the flavor and nutrient stimuli are mixed together in solution and when the nutrient US is presented intragastrically while the animal consumes the flavor CS. One of the interesting properties of this form of learning is that flavor preferences that result from these procedures have been shown to be extremely resistant to extinction. For example, Elizalde and Sclafani (1990; see also Drucker, Ackroff, \& Sclafani, 1994) demonstrated in rats that a preference was established for a flavor stimulus $(\mathrm{CS}+)$ paired with intragastric infusions of Polycose relative to a second flavor stimulus $(\mathrm{CS}-)$ paired with intragastric water infusions. Moreover, the preference for the nutrient-paired flavor did not diminish over 28 subsequent $\mathrm{CS}+$ versus CSchoice tests conducted under extinction conditions (i.e., without infusions).

Similar findings were reported more recently by Harris, Shand, Carroll, and Westbrook (2004) and by Albertella and Boakes (2006). In the Harris et al. (2004) study, thirsty rats initially consumed an almond flavor CS mixed in solution with a $4 \%$ sucrose US. In 20 subsequent choice tests pitting the flavor CS against plain water, the conditioned preference for the almond flavor CS was maintained at equally high levels.

In spite of the difficulty that investigators have reported in extinguishing a conditioned flavor preference, more precise measures of learning have been used to re- veal that extinction is not without effect in this paradigm. In particular, Delamater (2007a; see also Harris et al., 2004) recently provided evidence to suggest that the association formed between the flavor CS and the specific sensory properties of an orally presented nutrient US are either weakened or masked by extinction. In one experiment, thirsty rats were trained with two flavor CSs, each mixed in solution with a nutrient US (e.g., 8\% sucrose). Subsequently, one of these CSs was presented repeatedly (i.e., extinguished) without the sucrose US. In one group of rats, the sucrose US was then devalued (by separate pairings with lithium chloride [ $\mathrm{LiCl}]$ induced illness), whereas a second group of rats experienced sucrose and $\mathrm{LiCl}$ on separate days. A choice between the two flavor CSs revealed a preference for the nonextinguished over the extinguished flavor CS in control subjects, for whom sucrose was still valuable, but subjects exposed to the sucrose-devaluation treatment strongly preferred the extinguished flavor to the nonextinguished flavor CS. These data are consistent with the view that the flavor cues had been associated with the specific sensory properties of sucrose (see also Delamater, Campese, LoLordo, \& Sclafani, 2006; Dwyer, 2005) and that extinction weakened or masked control by this association to result in a preference for the nonextinguished or extinguished CS, depending on whether specific sensory properties of sucrose were valuable or not, respectively.

Another procedure sometimes thought to result in extinction-like effects is reversal learning (Delamater, 2007b). In a normal reversal task, one of two stimuli is first

A. R. Delamater, andrewd@brooklyn.cuny.edu 
paired with reinforcement, whereas the other is not, but these reward contingencies are switched in a second phase. Although numerous studies have revealed that animals' behavior is clearly sensitive to the change in reinforcement contingencies (for an example in flavor-preference learning, see Elizalde \& Sclafani, 1990), studies have not directly examined the effects of reversal training on the status of the sensory-specific associations learned first.

A second type of reversal task is one in which two different CSs are each paired with different USs initially, but then the specific CS-US combinations are switched during a reversal phase. To our knowledge, this sort of problem has not been extensively studied but may be of special interest in flavor-preference learning, because questions regarding the status of sensory-specific associations can be readily addressed using US devaluation procedures. For example, it is not known in this procedure whether during reversal learning the sensory-specific associations acquired initially might be undermined in some fashion. Several studies have found that stimuli (or responses) associated with two distinctive USs in sequential phases of the experiment become controlled by the first- and second-learned associations equally well (e.g., Delamater, 1996; Rescorla, 1992, 1995, 1997). These results suggest that specific associations between the CS and the sensory properties of the US can survive procedures in which a stimulus is paired with an alternative US and no longer paired with the US with which it was trained initially. It is noteworthy, however, that these reports used learning paradigms (conditioned magazine approach and instrumental training) quite different from flavor-preference conditioning. Since evidence of weakened or masked control by sensory-specific associations has been found in the flavor-preference paradigm (Delamater, 2007a; Harris et al., 2004) but not in other appetitive learning paradigms (e.g., Delamater, 1996; Rescorla, 1996), it seems possible that flavor-preference reversal learning might also produce effects similar to extinction.

In the present study, we explored this possibility. We report data from two experiments in which we used a USdevaluation procedure in an effort to assess the relative strengths of sensory-specific associations learned during an initial phase, as well as during a reversal phase.

\section{EXPERIMENT 1}

The first experiment was designed to test whether second-learned associations will diminish control by firstlearned associations in a flavor-preference reversal procedure. The experimental design is shown in Figure 1A. Two flavor CSs (almond and banana) were each associated with different nutrient USs (sucrose and Polycose) during an initial learning phase, but these associative relations were reversed in a second phase. Subsequently, one of the USs was devalued prior to tests given between the two flavor CSs. If the associations learned first and second did not differ in their relative strengths (see Delamater, 1996; Rescorla, 1992, 1995, 1997), we would expect subjects not to prefer one flavor CS over the other during a choice test conducted after one of the nutrients had been devalued. However, if the associations learned first were subsequently extinguished during the reversal phase (Delamater, 2007a), we would expect subjects to avoid the flavor CS that was associated most recently with the nutrient US that was devalued prior to the test and to prefer the flavor CS that was initially associated with this US.

\section{Method}

Subjects. The subjects were 24 Long Evans rats (12 male and 12 female), weighing 356-402 g (males) and 274-312 g (females) at the start of the experiment. The subjects were bred at Brooklyn College and derived from rats obtained from Charles River Laboratory. The subjects were not naive: They had previously participated in a Pavlovian conditioning task using visual and auditory stimuli, where pellets and sucrose $(0.1 \mathrm{ml}, 20 \%)$ were used as reinforcers. The rats had no prior exposure to the flavor CSs used in the present study. The subjects were individually housed in stainless steel cages $(24 \times 18 \times 17.5 \mathrm{~cm})$ and maintained on a 14:10-h light:dark cycle. Food chow was available ad lib throughout the study, but fluids were restricted to two 15-min drinking sessions per day, which were always $5 \mathrm{~h}$ apart, starting $4 \mathrm{~h}$ after the lights came on in the colony rooms. All sessions were conducted in the rats' home cages.

Solutions. The solutions were $8 \%$ Polycose (Ross Nutrition Laboratories) and $8 \%$ sucrose (pure Domino sugar) nutrients mixed in solution with $1 \%$ McCormick's Imitation flavor extracts (banana and almond). Intake of these solutions was measured by weighing all solutions before and after each session. The intake amounts were measured to the nearest $0.1 \mathrm{~g}$.

The solutions used during devaluation and nutrient testing consisted of nutrients presented in the absence of the flavor CSs. The

A

Design of Experiment 1:

\begin{tabular}{c|c|c|c|c} 
Acquisition & Reversal & US Devaluation & Flavor Test & Nutrient Test \\
\hline CS1 + US1 & CS1 + US2 & US1 $\rightarrow$ LiCl & CS1 vs. CS2 & US1 vs. US2 \\
CS2 + US2 & CS2 + US1 & US2- & &
\end{tabular}

B Design of Experiment 2:

\begin{tabular}{c|c|c|c|c|c|c|c} 
& Acquisition & Reversal & Delay & US Devaluation & Flavor Test & Nutrient Test & Flavor Test \\
\cline { 2 - 7 } Group Immediate & CS1 + US1 & CS1 + US2 & 1 Day & US1 $\rightarrow$ LiCl & CS1 vs. CS2 & US1 vs. US2 & CS1 vs. CS2 \\
\cline { 1 - 1 } & Group Delayed & CS2 + US2 & CS2 + US1 & 21 Days & US2- & &
\end{tabular}

Figure 1. Designs for Experiment 1 (A) and Experiment 2 (B). The conditioned stimuli (CSs) were distinct flavor cues (almond and banana), and the unconditioned stimuli (USs) were different nutrients (10\% sucrose and $10 \%$ Polycose). The + indicates that the flavor and nutrient were mixed in solution. The $\rightarrow$ indicates that the nutrient preceded and was paired with $\mathrm{LiCl}$. The - indicates that the nutrient was not paired with $\mathrm{LiCl}$. 
solutions used during the flavor-preference assessment tests consisted of the flavor CSs presented without the nutrient USs. The LiCl solution used during the devaluation phase was $0.3 \mathrm{M}$ injected intraperitoneally in the amount of $1 \%$ of the subject's body weight.

Acquisition and reversal. The subjects were initially given 3 days of 15 -min water drinking sessions presented twice daily with a 5-h separation for them to get used to the procedure. During the first phase of the experiment (Days 1-8), the subjects were given two flavor + nutrient solutions, each in one of the 15-min drinking sessions on each day. Half of the subjects (6 males and 6 females) received one set of pairings (e.g., almond + sucrose and banana + Polycose) and the other half ( 6 males and 6 females) received the other set (almond + Polycose and banana + sucrose). During the reversal phase (Days 9-16), each flavor + nutrient combination was reversed. Thus, each subject was trained with one flavor cue paired first with sucrose and then with Polycose (Fsp), and a second flavor cue paired first with Polycose and then with sucrose (Fps). All solutions were counterbalanced for time of day with each flavor-nutrient pair being presented once a day. The experiment was conducted 6 days per week. On the 7 th day, the subjects received two 15 -min sessions of tap water, which were presented at the same times as the solutions on other days.

Devaluation. Beginning on the morning following the last day of the reversal phase, the subjects were exposed to a selective US devaluation procedure over three 2-day cycles (Days 17-22). On the 1 st day of each cycle (Days 17, 19, and 21), all subjects were given sucrose, and, on the 2nd day of each cycle (Days 18, 20, and 22), all were given Polycose to drink. All nutrients were presented in the absence of the flavors. Half of the subjects ( 6 males and 6 females) were injected intraperitoneally with $\mathrm{LiCl}(0.3 \mathrm{M}, 1 \%$ body weight $)$ immediately after their consumption of sucrose, and the other half of the subjects ( 6 males and 6 females) were injected intraperitoneally after they consumed Polycose. Half of the subjects that were sucrose devalued came from the subgroup that received almond + sucrose and banana + Polycose pairings in the first phase, and the other half came from the alternate subgroup. The same was true for the Polycose-devalued group. In the evening session, the subjects were given tap water to drink for $15 \mathrm{~min}$ in their home cages. The presentation of nutrients took place at the same time as the morning session did during the acquisition and reversal phases and lasted $15 \mathrm{~min}$.

Testing. On the morning following the last day of the devaluation phase, the subjects received 1 day of two-bottle choice test training. The subjects were given two bottles, each containing tap water, one presented on the right side and one on the left side of the front of the cage, with spouts separated by approximately $3 \mathrm{~cm}$. In the evening session, all subjects were given tap water to drink for $15 \mathrm{~min}$ from a single bottle in their home cages.

Starting on the following morning, all subjects were given a flavor-preference test, which pitted almond against banana (counterbalanced for side). The rats were given $15 \mathrm{~min}$ of water in the evening session. This test was repeated on the following day, with the positions of the flavors on the cages counterbalanced across days.

The day after the completion of the flavor testing, the subjects were given 1 day of nutrient-preference testing, during which half of the subjects received the sucrose solution on the left side and the Polycose solution on the right side of the cage. The nutrient testing took place in the morning session at the usual time.

\section{Results}

Intakes of the two flavor + nutrient solutions did not differ throughout the acquisition or reversal phases and averaged approximately $15 \mathrm{~g}$. A three-way ANOVA examining the effects of flavor (Fsp vs. Fps), phase (acquisition vs. reversal), and day (1-8) did not reveal any significant main effects or interactions.

By the end of nutrient-devaluation training, subjects consumed less of the nutrient that was paired with $\mathrm{LiCl}$ than of the other nutrient. Separate cycle $\times$ solution ANOVAs using a pooled error term revealed significant interactions for the sucrose-devalued subjects $[F(1,22)=$ $36.17, p<.05]$ and for the Polycose-devalued subjects $[F(1,22)=47.04, p<.05]$, indicating that there were selective reductions in intake of the devalued nutrients over cycles.

The data of most interest came from the flavorpreference tests. These data, collapsed across the two flavor tests, are plotted in Figure 2A. Preliminary analyses did not reveal significant main effects or interactions involving sex, so the data were collapsed across this factor. The results illustrate that subjects avoided the flavor most recently paired with the devalued nutrient (i.e., Fps in sucrose-devalued subjects and Fsp in Polycose-devalued subjects). A two-way ANOVA yielded a significant flavor $($ Fsp vs. Fps $) \times$ devaluation (sucrose vs. Polycose)
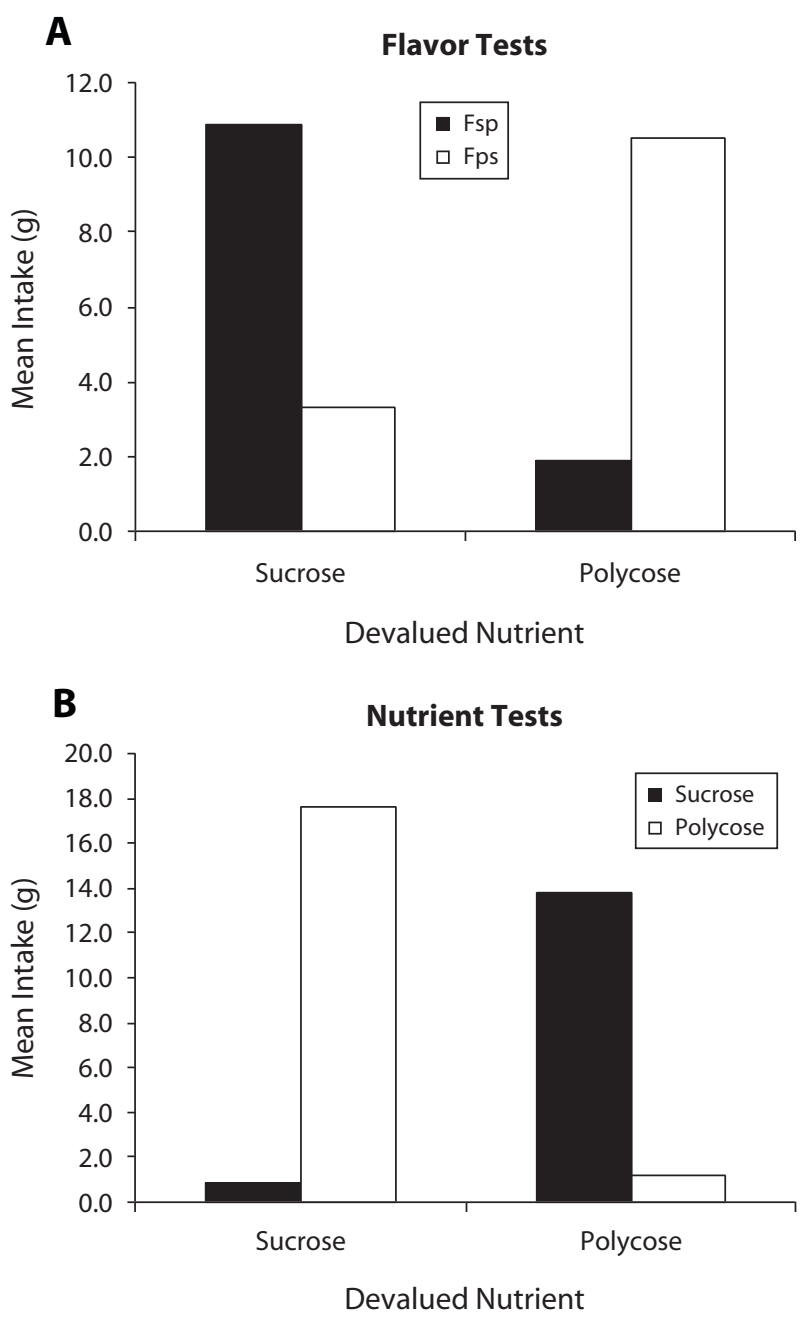

Figure 2. Flavor (A) and nutrient (B) test results from Experiment 1. Illustrated are the mean intakes of Fsp (flavor paired with sucrose during the acquisition phase and Polycose during the reversal phase) and Fps (flavor paired with Polycose during acquisition and sucrose during reversal) in the flavor tests (A) and of sucrose and Polycose in the nutrient tests $(B)$ in sucrose- and Polycose-devalued subjects. 
interaction $[F(1,22)=15.14, p<.05]$. Follow-up tests revealed a significant main effect of flavor in the sucrosedevalued group $[F(1,11)=7.08, p<.05]$ and in the Polycose-devalued group $[F(1,11)=9.37, p<.05]$.

The results of the nutrient tests are illustrated in Figure 2B. The sucrose-devalued subjects selectively avoided sucrose, whereas the Polycose-devalued subjects selectively avoided Polycose. A two-way ANOVA assessing the effects of devalued nutrient (sucrose vs. Polycose) and solution consumed (devalued vs. not devalued) yielded a significant main effect of solution $[F(1,22)=205.90$, $p<.05]$.

\section{Discussion}

The results from this experiment demonstrate that subjects avoided the flavor that was most recently associated with the devalued nutrient and, thus, preferred the flavor that was initially associated with the devalued nutrient but then subsequently associated with the nondevalued nutrient. This finding is consistent with those reported by Delamater (2007a) and suggest that pairing the flavor CSs with alternative nutrient USs in some way extinguished the originally learned associations. It may be noted that the results are not in total agreement with those reported by Delamater (1996) and Rescorla (1992, 1995, 1997), who failed to find a reduction in control by the originally acquired associations after the stimulus (or instrumental response) had been associated with a different US. However, there were many procedural differences between those experiments and the flavor-conditioning task used here that could account for this discrepancy.

Nevertheless, a fundamental question remains regarding the nature of the extinction effect found here. Does this effect reflect actual weakening of the originally acquired associations, or does the effect reflect the possibility that associations acquired during the reversal phase merely mask those acquired originally?

One of the primary sources of evidence to favor the second view comes from studies of spontaneous recovery, in which it has been repeatedly found that extinguished conditioned responses (CRs) reemerge when the stimulus is tested after a recovery interval (see, e.g., Rescorla, 2004). To our knowledge, it is not known whether associations acquired in the first phase of a flavor-preference reversal experiment will reemerge when subjects are tested after a delay, but there are data from other paradigms that are suggestive of this possibility (e.g., Bouton \& Peck, 1992; Lipatova, Wheeler, Vadillo, \& Miller, 2006; Urushihara, Wheeler, \& Miller, 2004). For example, Bouton and Peck used an appetitive-to-aversive (or aversive-to-appetitive) transfer procedure to study this question. An auditory CS was first paired with food in Phase 1 and then with shock in Phase 2. When tested immediately following Phase 2, subjects displayed aversive fear CRs, but, when tested following a 28-day retention interval, these were replaced by appetitive CRs characteristic of what was learned initially. These results suggest that originally established associations can recover over a retention interval in this paradigm. Furthermore, time-dependent effects have been reported in conditioned flavor aversion procedures using both latent inhibition (Aguado, Symonds, \& Hall, 1994; Lubow \& De la Casa, 2002) and spontaneous recovery designs (Rosas \& Bouton, 1996, 1998), and so recovery of Phase 1 learning might be expected to occur in the present circumstances as well. The next experiment investigates this possibility.

\section{EXPERIMENT 2}

The goal of this experiment was to determine whether the first-learned associations in a flavor-reversal procedure might recover to control preferences expressed during a test conducted after a delay following the reversal phase. In order to assess this question, the design illustrated in Figure 1B was employed.

The acquisition and reversal phases were identical to those used in Experiment 1. Thereafter, half of the subjects received selective nutrient devaluation the day following reversal training (Group Immediate) - as those in Experiment $1 \mathrm{did}$ - and the other half of the subjects received nutrient devaluation 3 weeks following reversal training (Group Delayed). Following devaluation, all subjects were given flavor tests and nutrient tests, similar to those conducted in Experiment 1.

If reversal learning weakens the initially formed associations, we predict that both of these groups should selectively avoid the flavor that was most recently associated with the devalued nutrient. However, if the second-learned associations merely mask control by the first-learned associations, the subjects in Group Delayed should recover their initially learned associations (see Bouton \& Peck, 1992; Lipatova et al., 2006; Urushihara et al., 2004) and could very well avoid the flavor that was initially paired with the devalued nutrient.

\section{Method}

Subjects. In Experiment 2, we used 32 Long Evans rats (16 males and 16 females), weighing 389-427 g (males) and 277-318 g (females) at the start of the experiment. The subjects were bred at Brooklyn College and derived from rats obtained from Charles River Laboratories. The subjects were not naive: They had previously participated in a Pavlovian conditioning task using visual and auditory stimuli, in which pellets and sucrose $(0.1 \mathrm{ml}, 20 \%)$ were used as reinforcers. The rats had no prior exposure to the flavor CSs used in the study. One of the subjects assigned to Group Delayed died during the acquisition phase; its data were excluded from all data analyses. The rats were maintained under the same conditions as those described in Experiment 1.

Solutions. Solutions used in this study were identical to those in Experiment 1.

Acquisition and reversal. Training was identical to that in Experiment 1, with the following exceptions: The acquisition and reversal phases for the two groups were staggered, so that all of the subjects were devalued and tested at the same time. More specifically, Group Delayed received the acquisition and reversal training first (Days 1-16). During this time, subjects in Group Immediate were given water to drink at the same time that subjects in Group Delayed were given their solutions. On Days 17-21, all subjects were given water for $15 \mathrm{~min}$, presented twice daily. Beginning on Day 22, Group Immediate began their Phase 1 training (Days 2242). Group Delayed subjects continued to receive water at the appropriate times.

Devaluation. The devaluation phase took place on Days 43-48 for subjects in both groups and was identical to that of Experiment 1. 
Testing. On the morning after the completion of the devaluation phase (Day 49), all subjects were given a two-bottle familiarization test with water in both bottles, followed $5 \mathrm{~h}$ later by water in one bottle in the afternoon session. Starting on the following day, all subjects were given a series of six flavor tests (Days 50-55) followed by two nutrient tests (Days 56-57), and then six more flavor tests (Days 58-63). During the first flavor-preference test, all of the subjects received the banana flavor on the left and the almond flavor on the right side of their cages. The flavors were presented on the opposite sides during Test 2 . In Tests 3-6, half of the subjects in each group received almond on the left and banana on the right. The rest of the subjects received the flavors on the opposite sides. Over Tests 3-6, the flavors were counterbalanced for side using an ABAB counterbalancing scheme. Following the first set of flavor tests, the subjects were given two nutrient tests, in which some subjects received sucrose on the left and Polycose on the right, and others received these nutrients on opposite sides. The following day, the nutrients were presented on the side opposite to that on the previous day. After the nutrient tests, the subjects were given six more flavor tests, where Tests 7,10 , and 11 were identical to Tests 3 and 5 , and Tests 8,9 , and 12 were identical to Tests 4 and 6 .

\section{Results}

The acquisition- and reversal-phase intake data from Experiment 2 were the same as in Experiment 1, with no significant differences in intake of the various flavor + nutrient compounds during acquisition or reversal. Once again, the average intake was close to $15 \mathrm{~g}$ in both groups. A four-way ANOVA analyzing the effects of flavor (Fsp vs. Fps), group (immediate vs. delayed), phase (acquisition vs. reversal), and day (1-8) revealed no significant main effects or interactions.

The data for the devaluation phase revealed that the rats in both Group Immediate and Group Delayed consumed less of the devalued nutrient than of the nondevalued nutrient by the end of nutrient-devaluation training. Significant cycle $\times$ solution (sucrose vs. Polycose) interactions were found for sucrose-devalued subjects $[F(2,14)=19.33, p<.05]$ and for Polycose-devalued subjects $[F(2,14)=28.66, p<.05]$ in Group Immediate, as well as for sucrose-devalued subjects $[F(2,14)=6.32$, $p<.05]$ and for Polycose-devalued subjects $[F(2,14)=$ $4.66, p<.05]$ in Group Delayed. This result suggests that subjects selectively reduced their intake of the devalued nutrient over cycles in both groups.

The data of main interest came from the flavorpreference tests and are illustrated in Figure 3. Preliminary analyses did not reveal any main effects or interactions involving sex, so the data were collapsed across this factor. The results for Tests $1-6$ were pooled together and are presented for each group in Figures $3 \mathrm{~A}$ and $3 \mathrm{~B}$.
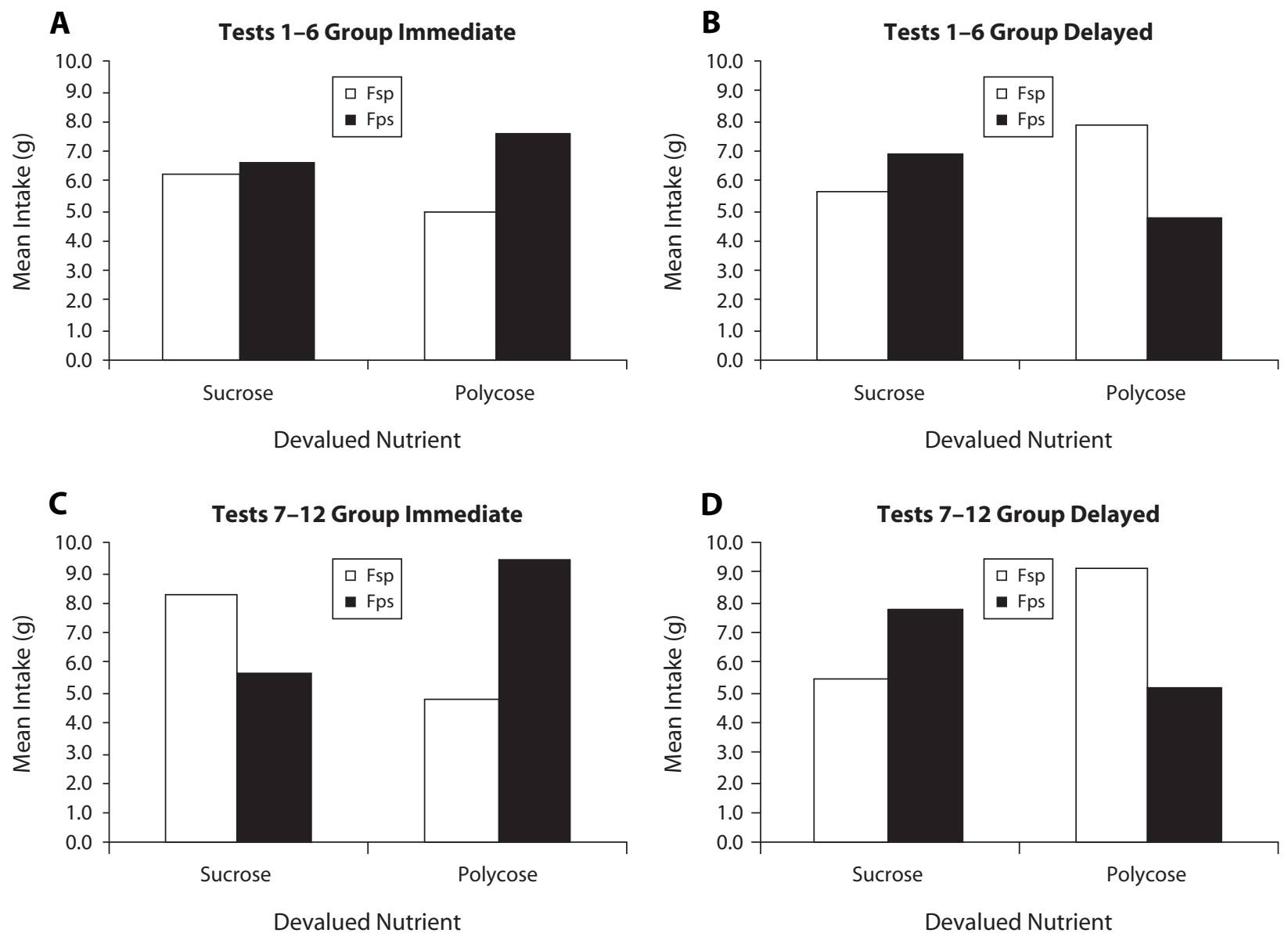

Devalued Nutrient

Devalued Nutrient

Figure 3. Flavor test results in Experiment 2: Mean intakes of Fsp (flavor paired with sucrose during the acquisition phase and Polycose during the reversal phase) and Fps (flavor paired with Polycose during acquisition and sucrose during reversal) during the flavor tests that preceded (A and B) or followed (C and D) the nutrient tests for Group Immediate (A and C) and Group Delayed (B and D). 
The results revealed that the Polycose-devalued subjects consumed less of the flavor that was recently paired with Polycose in Group Immediate but more of the flavor that was most recently paired with Polycose in Group Delayed. In neither group did the sucrose-devalued subjects exhibit any preferences.

A three-way ANOVA over these data yielded a significant flavor (Fsp vs. Fps) $\times$ devaluation (sucrose vs. Polycose) $\times$ group (immediate vs. delayed) interaction $[F(1,27)=4.91, p<.05]$. Follow up tests yielded a significant flavor $\times$ devaluation interaction for Group Delayed $[F(1,13)=4.94, p<.05]$ but no such interaction for Group Immediate $[F(1,14)=1.01, p>.05]$. Further tests for Group Delayed revealed a significant main effect of flavor in the Polycose-devalued subjects $[F(1,13)=6.85$, $p<.05]$, suggesting that these subjects avoided the flavor that was initially paired with Polycose and drank more of the flavor that was initially paired with sucrose. However, the sucrose-devalued subjects did not show any significant preference.

The data from Flavor Tests 7-12 were also pooled together and are presented in Figures 3C and 3D. More in line with the results from Experiment 1, Group Immediate subjects consumed less of the flavor that was recently paired with the devalued nutrient. This was true for both sucroseand Polycose-devalued subjects in this group. In contrast, Group Delayed subjects consumed less of the flavor that was initially paired with the devalued nutrient. This was also true for both sucrose- and Polycose-devalued subjects. These data were analyzed using a three-way ANOVA that revealed a significant flavor $\times$ group $\times$ devaluation interaction $[F(1,23)=4.33, p<.05]$. Further tests revealed a significant flavor $\times$ devaluation interaction for both Group Immediate $[F(1,14)=15.90, p<.05]$ and Group Delayed $[F(1,13)=5.60, p<.05]$. Subsequent tests in Group Immediate yielded significantly lower intake of the flavor that was recently paired with the devalued nutrient for sucrose-devalued subjects $[F(1,14)=5.26, p<.05]$ and for Polycose-devalued subjects $[F(1,14)=16.38, p<$ $.05]$. Conversely, the results for Group Delayed revealed lower intakes of the flavor that was initially associated with the devalued nutrient for the sucrose-devalued subjects $[F(1,13)=4.97, p<.05]$, as well as for the Polycosedevalued subjects $[F(1,13)=14.69, p<.05]$.

The nutrient-preference test results are illustrated in Figure 4 . These tests were conducted after the completion of the first set but before the second set of the flavorpreference tests. The results showed that the subjects in both groups strongly preferred the nondevalued nutrient over the devalued nutrient. A group (immediate vs. delayed) $\times$ devaluation (sucrose vs. Polycose) $\times$ solution (devalued vs. not devalued) ANOVA yielded a significant main effect of solution $[F(1,27)=119.60, p<.05]$ with no other main effects or interactions. These data suggest that subjects displayed a selective aversion to the devalued nutrient.

\section{Discussion}

The results for Group Immediate largely replicate those found in Experiment 1. The subjects in this group avoided the flavor that was most recently associated with the devalued nutrient. However, the subjects in Group Delayed avoided the flavor that was initially associated with the devalued nutrient. Both of these effects were more obviously detected in the set of flavor tests conducted after the nutrient tests.

The conclusions that subjects' performance during testing reflected their most recently learned associations when tested immediately after reversal learning and the initial associations when tested after a retention interval following reversal learning are consistent with the previous findings of Bouton and Peck (1992), Lipatova et al. (2006), and Urushihara et al. (2004). However, one unexpected finding in the present study was that these effects appeared weaker in sucrose-devalued than in Polycosedevalued subjects in the first set of tests. One explanation for this finding is that all of the subjects had prior experience with sucrose in a Pavlovian-to-instrumental transfer experiment that took place before the ones described in this
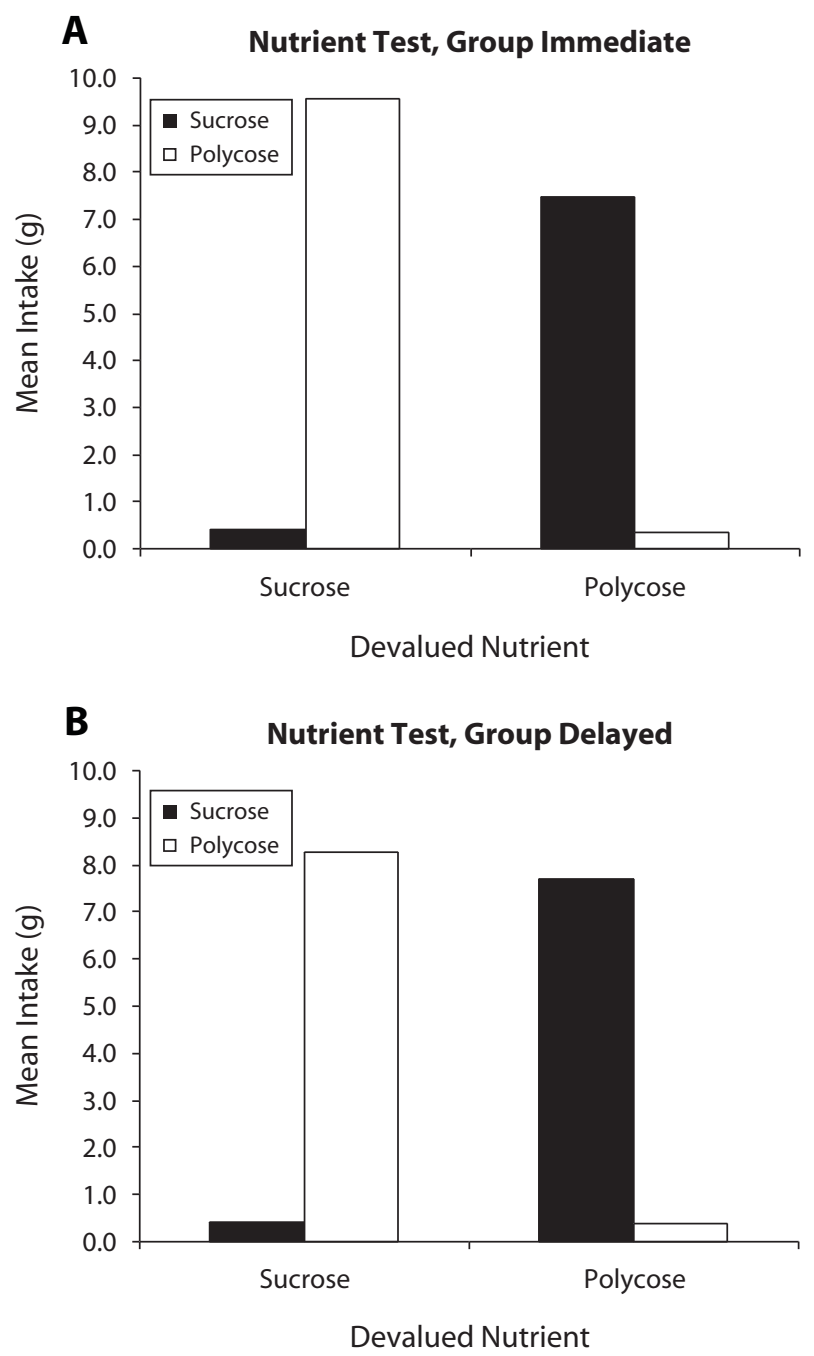

Figure 4. Nutrient test results in Experiment 2: Mean intakes of sucrose and Polycose nutrients in sucrose- and Polycose-devalued subjects in Group Immediate (A) and Group Delayed (B) in twobottle nutrient choice tests. 
study. It is possible that this prior experience with sucrose might have interfered with the formation of a sensoryspecific flavor-sucrose association during training. The US preexposure effect is a well-documented phenomenon shown to occur in a variety of learning paradigms (e.g., Cappell \& Le Blanc, 1977; Randich \& LoLordo, 1979), including flavor-preference learning (Harris, Gorissen, Bailey, \& Westbrook, 2000). It seems possible that sucrose preexposure might have retarded the development of a flavor-sucrose association in the present experiment, resulting in a somewhat weaker devaluation effect in our first set of tests with sucrose than in those with Polycose. Whether or not this is true, it is also noteworthy that the sucrose-devaluation effect in both groups was stronger when tested after the nutrient tests. Further work will be required to determine whether US preexposure and/or nutrient testing played an important role in the effects reported here. Other preliminary work conducted in our lab after these studies did not provide support for the possibility that nutrient testing, per se, plays a significant role.

\section{GENERAL DISCUSSION}

The results from the present experiments indicate that subjects acquire sensory-specific associations during both phases of a flavor-preference reversal learning task and that which association governs performance depends on how soon after reversal learning subjects are devalued and tested. In Experiment 1, we demonstrated that devaluing the US soon after reversal learning results in subjects' selectively avoiding the flavor CS that was most recently associated with that US. However, in Experiment 2, we observed this effect again but also found that subjects avoided the flavor CS that was initially associated with the US subsequently devalued if that devaluation treatment occurred 3 weeks after reversal learning. Apparently, both the original and reversed associations were learned and could control performance, depending on time since reversal. These findings have implications for our understanding of how the basic US devaluation effect works and also for the nature of reversal and extinction learning.

In the present experiment, we used a US devaluation technique to assess the status of sensory-specific flavornutrient associations at different times following reversal learning. Prior studies have used this technique to illustrate that, in flavor-preference conditioning, the flavors can, indeed, form an association with some specific sensory property of the nutrient (Delamater et al., 2006; Dwyer, 2005; Harris et al., 2004). However, there are at least two different ways in which a US devaluation treatment could reduce preference for the associated flavor CS. First, if some specific sensory property of the nutrient is devalued after conditioning, the flavor CS should be avoided at test to the extent that it associatively activates a representation of those devalued sensory features of the nutrient. This mechanism could account for the present results if it were additionally assumed that which nutrient the flavor CS associatively activates depends on how soon after reversal training the test occurs. If the relative strengths of the first- and second-learned associations change over time, this mechanism could produce the other pattern of results seen in Experiment 2.

A second way in which US devaluation could work is through what some authors have referred to as mediated conditioning (Hall, 1996; Holland, 1981). In particular, at the time of US devaluation, it is possible that the nutrient associatively activates a representation of the flavor CS with which it is most strongly associated. Presumably, this would be the most recent associate of the nutrient when devaluation occurs soon after reversal training but the initial associate of the nutrient when devaluation occurs 3 weeks following reversal training. Accordingly, a direct association could then be formed between the evoked representation of the CS flavor and $\mathrm{LiCl}$-induced illness when it occurs. This mechanism could apply equally to the present results if it were assumed both that specific associations were learned during the acquisition and reversal phases and that their relative strengths varied with time.

In the present study, it is possible that both of the mechanisms discussed above could apply, especially since we employed simultaneous-conditioning procedures. In such a case, we would expect the CS flavor to associate with the nutrient US and the nutrient US to associate with the CS flavor. We have no reason to suppose that the directionality of these associations would be biased one way or the other. However, it remains possible that, if a sequentialconditioning procedure were used (with the flavor CS preceding the nutrient US), in which the flavor-nutrient association is presumably stronger than any nutrientflavor association, a different pattern of results could be obtained.

It should be noted that, since both of these mechanisms predict the same outcome under more normal circumstances not involving reversals, it has been difficult to distinguish experimentally between these two mechanisms. However, use of a reversal task may be especially helpful in discriminating between these two accounts. Suppose that, instead of imposing a delay between reversal training and devaluation, the delay were imposed between devaluation training and test. In such an experiment, the mediatedconditioning account would anticipate that imposing a delay should have no effect, because the mediated learning should be the same, given that the reversal-to-devaluation interval would be the same. However, the first mechanism described above would anticipate the same pattern of results as those reported in Experiment 2, because the flavor CSs would still retrieve representations of different nutrients, depending on time since reversal training. It remains to be determined which of these possible mechanisms was most likely at work in the present circumstances, but, at the very least, the present methods offer a way in which these questions can be approached in future studies.

Another noteworthy feature of the present results is that they support a growing consensus that extinction-like effects entail some sort of masking process and not unlearning (see, e.g., Bouton, 2004; Bouton, Westbrook, Corcoran, \& Maren, 2006; Delamater, 2004). In Experiment 1, we observed that subjects' flavor preferences were based on their most recently acquired associations, whereby they avoided the flavor CS most recently associated with the devalued 
nutrient. This result could have occurred either because the associations during the reversal phase replaced the associations learned first or because they transiently masked the associations learned first. Because, in Experiment 2, we observed recovery of the initially acquired associations in Group Delay, this result would favor the masking view and support the notion that the initially acquired associations were not weakened by reversal.

However, it may have come as a surprise to some that the recovered Phase 1 associations in Experiment 2 seemed to supplant control by the reversal-phase associations. Earlier work has demonstrated that, when a stimulus (or instrumental response) is paired with multiple outcomes in successive phases, the associations seem to contribute equally to performance during a test (e.g., Delamater, 1996; Rescorla, 1992, 1995, 1997). Although recovery similar to that in our results has been observed to occur in other paradigms (Bouton \& Peck, 1992; Lipatova et al., 2006; Urushihara et al., 2004), why the recovered Phase 1 associations should dominate control by the reversal-phase associations is a finding in need of explanation. One possibility is that, although the reversal phase associations could have masked control by the initial associations, the initial associations could have proactively interfered with learning the reversal-phase associations. Once the masking process was removed (after a 3-week delay), the difference in relative strengths of the initial- and reversal-phase associations was revealed. A second possibility is that, as was suggested by Swartzentruber and Bouton (1992) and Nelson (2002), information acquired in a second phase of training is more subject to contextual influences than is information acquired first. If time since reversal in the present studies can be considered a contextual cue (e.g., Bouton, 1991, 2004), this sort of analysis could readily apply to the present circumstances. Regardless of which of these alternatives is correct, this discussion underscores the importance of determining when one might expect to observe control exerted by associations learned first, second, or as some mixture of the two.

One of the motivations for carrying out the experiments reported here was to examine a potential parallel between reversal learning and extinction. In both cases, contingencies present during an initial phase are removed during a second phase, and, thus, we might expect similar extinctionlike processes to occur in both cases. Our results are reminiscent of spontaneous recovery (see, e.g., Rescorla, 2004), one of the hallmark features of extinction. Finding this sort of recovery in the context of reversal learning is, therefore, interesting; however, it remains to be determined whether a similar sort of recovery would occur from extinction in a flavor-preference paradigm. Delamater (2007a) reported that sensory-specific associations are either weakened or masked by extinction in this paradigm. The present data suggest that extinction most likely masked control by the original association, but further work is needed to verify whether this was, indeed, the case.

The present data are also of interest from the perspective of recent work on the neural mechanisms of reversal and extinction learning. Schoenbaum and colleagues have identified the orbitofrontal cortex and basolateral amygdala as two important structures in reversal learning (for a review, see, e.g., Schoenbaum, Saddoris, \& Stalnaker, 2007). Schoenbaum, Chiba, and Gallagher (1999), for instance, identified cells within these structures that adjust their firing patterns to reflect the current contingencies during an instrumental reversal learning task. In other words, whereas some cells during initial training fired (e.g., in response to an $\mathrm{S}+$ but not to an $\mathrm{S}-$ ), these very same cells responded (especially in the basolateral amygdala) during the reversal phase only to the former $\mathrm{S}-$ (but current $\mathrm{S}+$ ). These results at the level of single-cell electrophysiology converge with ours at the level of behavior, but our results go on to suggest that the original associations controlling behavior can recover over a retention interval. It remains to be seen how the brain accomplishes this recovery.

One clue comes from studies examining the neural mechanisms of extinction. The infralimbic (IL) region of the ventromedial prefrontal cortex is thought to play an important role in extinction (for a recent review, see Quirk \& Mueller, 2008). Recent research has demonstrated that IL lesions result in more rapid response recovery 1 day following extinction in both aversive (Santini, Muller, \& Quirk, 2001) and appetitive (Rhodes \& Killcross, 2004, 2007) paradigms. It is possible, therefore, that the IL region plays a role in suppressing original learning in a reversal procedure as well. It remains to be seen, however, whether IL lesions might similarly influence the apparent spontaneous recovery of original learning seen after reversals in the present circumstances. Indeed, the notion that the neural mechanisms underlying extinction and reversal learning may involve overlapping circuits has, to our knowledge, not been explored.

In summary, in the present experiments, we addressed the question of whether flavor-preference reversal learning displays properties similar to extinction. We observed that preferences based on sensory-specific flavor-nutrient associations were controlled by associations learned during the reversal phase when testing occurred immediately after reversal training but that they were controlled by associations learned initially when testing occurred 3 weeks after reversal learning. These results suggest that initially learned sensory-specific associations are not weakened by the associations acquired during a reversal phase. Instead, these initially acquired associations seem to spontaneously recover over a delay. In this sense, the learning that occurs when a previously conditioned stimulus is nonreinforced and when it is reinforced with a different reinforcing outcome than that used originally may involve similar underlying mechanisms.

\section{AUTHOR NOTE}

This research was supported by NIMH Grant 065947 awarded to A.R.D. The authors gratefully acknowledge Leib Litman for his constructive input on these data. Correspondence concerning this article should be addressed to A. R. Delamater, Psychology Department, Brooklyn College, 2900 Bedford Ave., Brooklyn, NY 11210 (e-mail: andrewd@brooklyn.cuny.edu).

\section{REFERENCES}

Aguado, L., Symonds, M., \& Hall, G. (1994). Interval between preexposure and test determines the magnitude of latent inhibition: Im- 
plications for an interference account. Animal Learning \& Behavior, 22, 188-194.

Albertella, L., \& Boakes, R. A. (2006). Persistence of conditioned flavor preferences is not due to inadvertent food reinforcement. Journal of Experimental Psychology: Animal Behavior Processes, 32, 386-395.

Bouton, M. E. (1991). Context and retrieval in extinction and in other examples of interference in simple associative learning. In L. Dachowski \& C. F. Flaherty (Eds.), Current topics in animal learning: Brain, emotion, and cognition (pp. 25-53). Hillsdale, NJ: Erlbaum.

Bouton, M. E. (2004). Context and behavioral processes in extinction. Learning \& Memory, 11, 485-494.

Bouton, M. E., \& Peck, C. A. (1992). Spontaneous recovery in crossmotivational transfer (counterconditioning). Animal Learning \& Behavior, 20, 313-321.

Bouton, M. E., Westbrook, R. F., Corcoran, K. A., \& Maren, S. (2006). Contextual and temporal modulation of extinction: Behavioral and biological mechanisms. Biological Psychiatry, 60, 352-360.

Cappell, H., \& Le Blanc, A. E. (1977). Parametric investigations of the effects of prior exposure to amphetamine and morphine on conditioned gustatory aversion. Psychopharmacology, 51, 265-271.

Delamater, A. R. (1996). Effects of several extinction treatments upon the integrity of Pavlovian stimulus-outcome associations. Animal Learning \& Behavior, 24, 437-449.

Delamater, A. R. (2004). Experimental extinction in Pavlovian conditioning: Behavioural and neuroscience perspectives. Quarterly Journal of Experimental Psychology, 57B, 97-132.

Delamater, A. R. (2007a). Extinction of conditioned flavor preferences. Journal of Experimental Psychology: Animal Behavior Processes, 33, 160-171.

Delamater, A. R. (2007b). The role of the orbitofrontal cortex in sensory-specific encoding of associations in Pavlovian and instrumental conditioning. In G. Schoenbaum, J. A. Gottfried, E. A. Murray, \& S. J. Ramus (Eds.), Linking affect to action: Critical contributions of the orbitofrontal cortex (Annals of the New York Academy of Sciences, Vol. 1121, pp. 152-173). New York: New York Academy of Sciences.

Delamater, A. R., Campese, V., LoLordo, V. M., \& Sclafani, A. (2006). Unconditioned stimulus devaluation effects in nutrientconditioned flavor preferences. Journal of Experimental Psychology: Animal Behavior Processes, 32, 295-306.

Drucker, D. B., Ackroff, K., \& Sclafani, A. (1994). Nutrientconditioned flavor preference and acceptance in rats: Effects of deprivation state and nonreinforcement. Physiology \& Behavior, 56, 701-707.

DWYER, D. M. (2005). Reinforcer devaluation in palatability-based learned flavor preferences. Journal of Experimental Psychology: Animal Behavior Processes, 31, 487-492.

Elizalde, G., \& SClafani, A. (1990). Flavor preferences conditioned by intragastric Polycose infusions: A detailed analysis using an electronic esophagus preparation. Physiology \& Behavior, 47, 63-77.

HALL, G. (1996). Learning about associatively activated stimulus representations: Implications for acquired equivalence and perceptual learning. Animal Learning \& Behavior, 24, 233-255.

Harris, J. A., Gorissen, M. C., Bailey, G. K., \& Westbrook, R. F. (2000). Motivational state regulates the content of learned flavor preferences. Journal of Experimental Psychology: Animal Behavior Processes, 26, 15-30.

Harris, J. A., Shand, F. L., Carroll, L. Q., \& Westbrook, R. F. (2004). Persistence of preference for a flavor presented in simultaneous compound with sucrose. Journal of Experimental Psychology: Animal Behavior Processes, 30, 177-189.

Holland, P. C. (1981). Acquisition of representation-mediated conditioned food aversions. Learning \& Motivation, 12, 1-18.

Lipatova, O., Wheeler, D. S., Vadillo, M. A., \& Miller, R. R.
(2006). Recency-to-primacy shift in cue competition. Journal of Experimental Psychology: Animal Behavior Processes, 32, 396-406.

Lubow, R. E., \& De La CASA, L. G. (2002). Superlatent inhibition and spontaneous recovery: Differential effects of pre- and postconditioning CS-alone presentations after long delays in different contexts. Animal Learning \& Behavior, 30, 376-386.

Nelson, J. B. (2002). Context specificity of excitation and inhibition in ambiguous stimuli. Learning \& Motivation, 33, 284-310.

Quirk, G. J., \& MuelLer, D. (2008). Neural mechanisms of extinction learning and retrieval. Neuropsychopharmacology, 33, 56-72.

Randich, A., \& LoLordo, V. M. (1979). Associative and nonassociative theories of the UCS preexposure phenomenon: Implications for Pavlovian conditioning. Psychological Bulletin, 86, 523-548.

RESCORLA, R. A. (1992). Associations between an instrumental discriminative stimulus and multiple outcomes. Journal of Experimental Psychology: Animal Behavior Processes, 18, 95-104.

ResCorla, R. A. (1995). Full preservation of a response-outcome association through training with a second outcome. Quarterly Journal of Experimental Psychology, 48B, 252-261.

Rescorla, R. A. (1996). Preservation of Pavlovian associations through extinction. Quarterly Journal of Experimental Psychology, 49B, 245-258.

ResCorla, R. A. (1997). Spontaneous recovery after Pavlovian conditioning with multiple outcomes. Animal Learning \& Behavior, 25, 99-107.

Rescorla, R. A. (2004). Spontaneous recovery. Learning \& Memory, 11, 501-509.

RHodes, S. E. V., \& Killcross, [A.] S. (2004). Lesions of rat infralimbic cortex enhance recovery and reinstatement of an appetitive Pavlovian response. Learning \& Memory, 11, 611-616.

Rhodes, S. E. V., \& Killcross, A. S. (2007). Lesions of rat infralimbic cortex enhance renewal of extinguished appetitive Pavlovian responding. European Journal of Neuroscience, 25, 2498-2503.

Rosas, J. M., \& Bouton, M. E. (1996). Spontaneous recovery after extinction of a conditioned taste aversion. Animal Learning \& Behavior, 24, 341-348.

Rosas, J. M., \& Bouton, M. E. (1998). Context change and retention interval can have additive, rather than interactive, effects after taste aversion extinction. Psychonomic Bulletin \& Review, 5, 79-83.

Santini, E., Muller, R. U., \& Quirk, G. J. (2001). Consolidation of extinction learning involves transfer from NMDA-independent to NMDA-dependent memory. Journal of Neuroscience, 21, 9009 $-9017$.

Schoenbaum, G., Chiba, A. A., \& Gallagher, M. (1999). Neural encoding in orbitofrontal cortex and basolateral amygdala during olfactory discrimination learning. Journal of Neuroscience, 19, 1876-1884.

Schoenbaum, G., Saddoris, M. P., \& Stalnaker, T. A. (2007). Reconciling the roles of orbitofrontal cortex in reversal learning and the encoding of outcome expectancies. In G. Schoenbaum, J. A. Gottfried, E. A. Murray, \& S. J. Ramus (Eds.), Linking affect to action: Critical contributions of the orbitofrontal cortex (Annals of the New York Academy of Sciences, Vol. 1121, pp. 320-335). New York: New York Academy of Sciences.

Swartzentruber, D., \& Bouton, M. E. (1992). Context sensitivity of conditioned suppression following preexposure to the conditioned stimulus. Animal Learning \& Behavior, 20, 97-103.

Urushinara, K., Wheeler, D. S., \& Miller, R. R. (2004). Outcome pre- and postexposure effects: Retention interval interacts with primacy and recency. Journal of Experimental Psychology: Animal Behavior Processes, 30, 283-298.

(Manuscript received July 22, 2008; revision accepted for publication October 20,2008.) 